\title{
Towards a Unified Business Strategy Language: A Meta-model of Strategy Maps
}

\author{
Constantinos Giannoulis ${ }^{1}$, Michael Petit ${ }^{2}$, and Jelena Zdravkovic $^{1}$ \\ ${ }^{1}$ Department of Computer and Systems Sciences \\ Stockholm University \\ Forum 100, SE-164 40 Kista, Sweden \\ ${ }^{2}$ PReCISE Research Center, Computer Science Department \\ University of Namur \\ Rue Grandgagnage 21, B-5000 Namur, Belgium \\ \{constantinos, jelenaz\} @dsv.su.se, \\ mpelinfo. fundp.ac.be
}

\begin{abstract}
Alignment between business strategies and the resources engaged ensuring their realization, has been a continuous concern of enterprises of all kinds in last few decades. Commonly, enterprises fail to establish the traceability from business strategies towards operational tasks carried by employees. From the requirements engineering perspective this problem leads also to a misalignment between business and IT assets. In this study, we argue that for communicating high-level intentions and strategies down to the operational perspective, i.e. tasks and resources, the core necessity is to have a rich and well-defined language for modeling business strategies. Such a language could be further utilized for facilitating formalizations and a constructive analysis of high-level business aspects of enterprises, as well for comparing and unifying existing intentional modeling languages from the business and requirements engineering domains. As a reference proposal for formalizing business strategies, we consider the well-established strategy maps [1] from the Management Information Systems community which provide textual concepts of strategy-related notions establishing causal relationships between them. We have set an effort to formalize strategy maps in the form of a meta-model, usage scenarios and constraints, providing a systematic basis for obtaining a unified language/ontology for business strategy modeling.
\end{abstract}

Keywords: Business strategy, strategy maps, goal modeling, meta-model.

\section{Introduction}

New business environments foster enterprises to streamline their organizational structures, activities and resources in a way to align them with long-term business visions and strategies.

In this effort, the major concern is to enable communicating high level strategies from executives toward employees, and from product and service offers toward concrete tasks [2]. A proposal concerning notions pertinent to capturing business 
strategies and their realizations comes from the work on strategy maps by Kaplan and Norton [1]. Briefly, the maps represent strategic blueprints of enterprises observed from several perspectives, i.e. from business visions and product offers down to internal capabilities and assets.

In the "modeling" community, business intentions and following strategies are typically conceptualized using goal-based languages, such as in Goal-Oriented Requirements Engineering (GORE) [3] with i* [4], KAOS [3], etc. as well as others coming from the business domain, such as the Business Motivation Model, BMM [5].

The major question in the previously described context concerns the capabilities of goal-oriented languages to capture end express the perspectives, the notions and their relations as mandated by strategy maps. As formal and as precise they are, being generic makes questionable their representational capabilities for business strategies as opposed to dedicated schemes such as strategy maps which provide rich expressiveness.

We aim to analyze, compare and unify goal modeling languages from the requirements engineering domain, which support formality, preciseness and are generic enough to capture any goal related aspect, with those from the business domain, which are rich in expressiveness and focus on business strategies. The overall objective is to leverage from both domains and obtain a rich and expressive language capable of capturing intentions and strategies from visionary to operational enterprise perspectives supporting mappings to current schemes practiced.

In order to define this rich language, we will use a systematic approach in which existing languages for modeling intentions are analyzed and evaluated with respect to their suitability to represent aspects relevant to business strategy formulations. Our approach will be based on ideas developed in the Unified Enterprise Modeling Language (UEML) approach whose objective is to create a framework for interoperability of enterprise modeling languages and to define "a core language for enterprise and IS modeling" [6]. The UEML approach has analyzed a number of existing modeling languages by mapping their constructs onto a common and well defined ontological base. By doing so, the understanding of concepts of existing languages was improved and it was possible to progressively grow a larger ontology containing well connected concepts by extending the ontological base.

For our research effort we will apply a similar approach with the aim to define a core language for business strategy modeling, by analyzing one by one existing languages allowing the representation of business goals cited earlier (BMM, $\mathrm{i}^{*}$, KAOS, etc.). For our starting point in this direction, we have to choose an ontological base that will serve as the basis for analysis of each language. In our case, we have chosen strategy maps as a starting ontological base because it is a widely used method to represent business strategies. Gradually, during the coming steps of our work we will examine other approaches that represent business strategies. Along with the gradual evolution of our ontological base we will be incrementally building relevant mappings of our core language to existing goal-based languages to provide applicability for real practice.

The first step in this research, which forms the scope of this paper, requires the conceptualization of strategy maps, as well as a clarification of possible usage scenarios, to provide a well-formed and systematic basis for further analysis of goalmodeling languages. 
The paper is structured as follows: Section 2 presents the related work on goal modeling languages, business strategy and alignment. Section 3 presents our contribution, starting from the strategy map template to its conceptualization to a meta-model. In Section 4, the conclusions and directions for further research are briefly discussed.

\section{Related Work}

\subsection{Goal Modeling}

Intentions and consecutive strategies are typically conceptualized using goal-based languages stemming from the requirements engineering domain as well as from the business domain. Languages in the former domain focus on formality and preciseness of systems to be developed, business goals, processes, etc. supporting organizational objectives, though significant variations exist. The latter focuses on expressiveness of business models for supporting business plans.

\subsubsection{Requirements Engineering Domain}

Within requirements engineering, the work of Kavakli and Loucopoulos [7] provide an evaluation-oriented overview of the most prominent GORE methods. Methods are grouped according to their role in requirements engineering along the four phases in the RE process: elicitation, negotiation, specification and validation. Additionally, the methods are distinguished in terms of formality as semi-formal and formal. Semiformal methods are imprecise and relations between elements are loosely defined, while they allow stakeholders to establish a shared view of the situation and set an agreeable frame around further analysis to be performed (e.g. GSN [8], GQM [9]). Formal methods lack on goal elicitation, simplicity and flexibility, while they are unambiguous, they support consistency as well as precise representation of goals and have strong semantics (e.g. i*, KAOS). Regardless of grouping, neither based on role nor on level of formality, none of the evaluated methods addresses adequately the complexity of goal analysis. Within an enterprise context, goal analysis relies primarily on stakeholders' interests making their involvement in the modeling process essential. Additionally, a lack of the methods and techniques to identify potential stakeholders, as well as to facilitate their cooperation is also acknowledged.

A more recent evaluation within GORE by Amyot et al [10] describes goal models using the Goal-oriented Requirement Language (GRL), which is part of the User Requirements Notation (URN). URN is a new Recommendation of the International Telecommunications Union, provides the first standard goal-oriented language. GRL integrates concepts of $i^{*}$ and the NFR Framework [11] along with a scenario notation included constitutes GRL, which is scalable and consistent in terms of representation of different view for the same goal model, thus supporting the views of various stakeholders. Additionally, GRL, based on the $i^{*}$ language, can capture the high-level business goals and the non-functional requirements for a stakeholder as well as possible alternatives for the achievement, and therefore, allowing the evaluation of trade-off analysis between alternatives. 


\subsubsection{Business Domain}

Goal-based languages from the business domain are focused on the expressiveness of business model and their relation to business plans.

The Business Motivation Model (BMM) is a proposed standard of the Object Management Group (OMG) - it is focused on business plans, including their motivating factors, elements and their interrelations. The main parts of the BMM are the Ends, the Means and the Influencers. The Ends capture enterprise goals and objectives; the Means captures enterprise strategies, tactics, rules and policies, which together represent what is an enterprise aiming at achieving. The Influencers capture the elements of business plans as well as assessments on their impact towards Means and Ends, which justifies the existence of each element of a business plan.

Another goal modeling approach is the formal framework of Popova and Sharpanskykh [12] which uses performance indicators to monitor the progress of an enterprise. Essential principle of this approach is the fact that "each organization exists for the achievement of one or more goals". Goals are clearly related to the performance indicators of an organization and a clear connection between them is established through goal structures. The approach allows goal classifications and different degrees of goal satisfaction but enforces a formal and unified representation of goal hierarchies, which makes them dependent to the modeler's perspective.

\subsection{Business Strategy}

Strategic planning is the process during which a strategy is defined by analyzing the current status of the enterprise and the competitive environment in which it resides. As pointed out in [13], citing Barney [14], three main types of approaches focusing on different aspects have been proposed. The "resource-based view" argues that the competitive advantage of a firm depends mainly on its distinctive unique capabilities, provided by its resources.

The "industrial organization perspective" (exemplified by the work of Porter) argues that the competitive advantage will result from a clear positioning of the firm with respect to its environment, described by the structure of the industry based e.g. on the five forces model of Porter. The positioning requires the choice of a strategic line among three possible ones (cost leadership, differentiation or focus) that will be implemented by carefully considering the required capabilities of internal processes of the value chain in terms of cost and quality.

The last perspective, the "Schumpeterian view of competition" centers on competitive innovation and rests on the idea that radical innovations can disrupt the industrial environment in which the firm operates, thereby giving opportunities to take an advantage over companies who's capability to innovate is lower.

A good strategic planning process commonly takes into account these three different but complementary views on the subject.

Strategy Maps and Balance Scorecards [15] have been proposed by Kaplan and Norton as a means to represent the strategy of a company in order to be able to communicate and to monitor the achievement of the strategic objectives. A strategy map serves as a mediator between the Mission, core Values, the Vision and the Strategy of an enterprise to the work performed. It is one of the rare frameworks providing means for visual representation of a strategy. Therefore, for all outlined features, we give it a particular importance in our work. 


\subsection{Alignment}

Several research works have proposed combining several languages in order to address the issue of aligning strategy and value proposition with related operational processes.

Within [16], $i^{*}$ is used for the representation of strategic goals, the value proposition model is described by business schematics and Role-Activity-Diagrams (RAD) describe processes.

The INSTAL method proposes a map-type to represent strategic intents and complementary process formalisms for business processes, integrated in a single meta-model [17]. Similarly, [18] puts forward a map-driven process modeling approach based on intentions and strategies capable of abstracting organizational goals and their achievement from detailed business processes.

In [19] and [20], the combination of $i^{*}$ for strategic goals modeling and e 3 value for value proposition description is proposed.

In [21], the combination of several goals languages (i*, BMM, KAOS) and value modeling ontology (e3value, REA, etc.) is analyzed and detailed rules for combination are proposed.

The $3 \mathrm{~g}$ framework for business-IT alignment proposed in [22] is based on multidisciplinary goal concepts and focuses on linking task goals to strategic business goals.

The study presented in [23] is close to ours using strategy maps and requirements engineering approaches for alignment, which are demonstrated through a mapping of constructs between $i^{*}$ and strategy maps.

\section{Modeling and Utilizing Strategy Maps}

The content of this section includes our effort on conceptualizing strategy maps into a well-defined meta-model. For the development of the meta-model we have conducted a comprehensive literature study on strategy maps [15] [24] [25], as well as on published outcomes of their implementation [1] [15] [24] [26] [27]. Given the limited formalisms of the strategy map proposal and the ambiguities found in numerous textual descriptions, we have considered and analyzed them all, to obtain a precise and complete formalization. The first part of this section gives an overview of strategy maps and an analysis of identified usage distinctions, while the second part presents the developed meta-model.

\subsection{Strategy Maps (SM)}

A strategy map is a general representation of the four organizational perspectives of the Balance Scorecard framework (BSC) [15]. According to [26], a BSC presents an organization's business activities through a number of measures typically from four organizational perspectives: financial, customer, internal, learning and growth, and provides a language to communicate priorities within an enterprise. A scorecard is considered balanced (BSC) due to the four perspectives that provide complete coverage of business processes, the time aspect covered in a bottom up manner suggesting that what lies on the bottom is the outcome of planning at the top and has 
taken place in the past. Additionally a scorecard is also considered balanced because it covers both the internal as well as the external aspects of an enterprise. Finally, a BSC is structured based on cause-effect links/assumptions who's monitoring and assessment is essential for identifying interdependencies across an enterprise.

A strategy map is a comprehensive representation of strategy that captures linkages of the four BSC perspectives in a cause-effect manner and facilitates executives in communicating direction and priorities across the enterprise on all levels, thus creating enterprise alignment. Kaplan \& Norton have proposed a template for strategy maps (figure below) representing how an organization can create value [24].

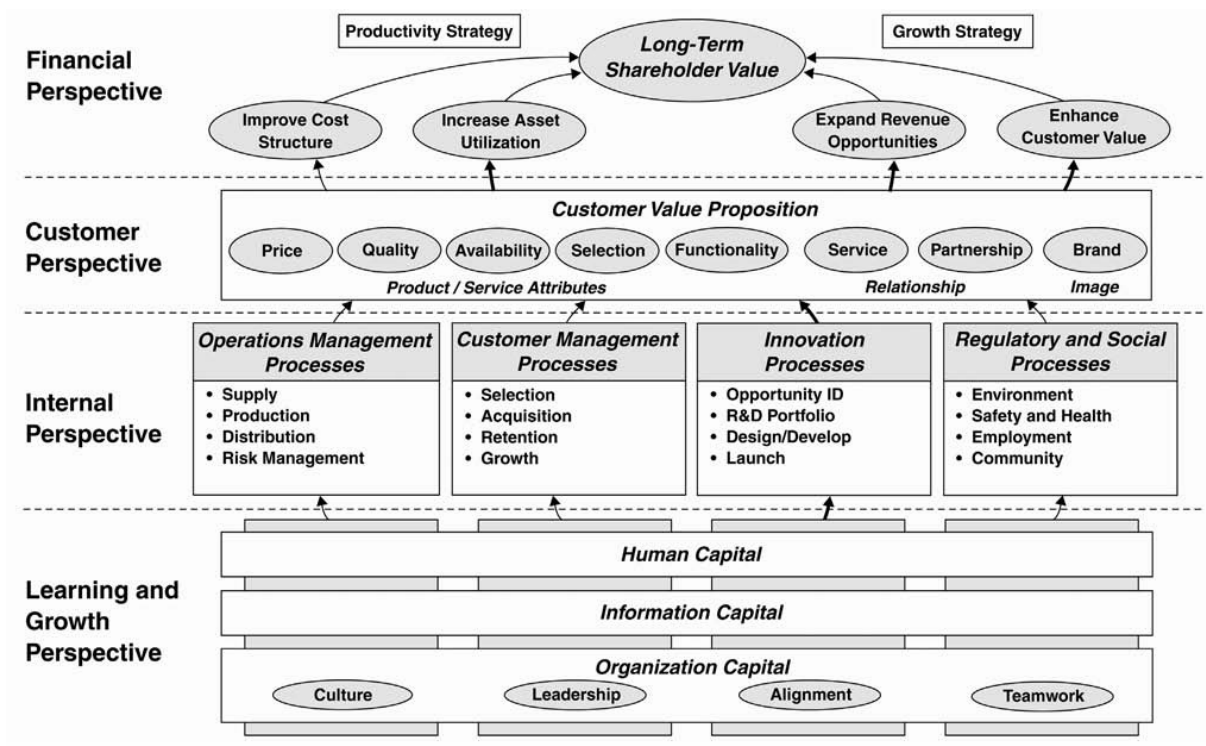

Fig. 1. The Strategy Map template [1] [15]

According to [24], a strategy map is based on five principles:

- Strategy balances long-term financial commitments aiming at profitable revenue growth and short-term financial commitments aiming at cost reductions and productivity improvements.

- Strategy is based on differentiated and clearly articulated customer value proposition.

- Value is created through focused, effective and aligned internal business processes grouped into four clusters: operations management, customer management, innovation and regulatory and social.

- Strategy consists of simultaneous, complementary themes highlighting the most critical processes supporting the customer value proposition.

- Strategic alignment determines the value and role of intangible assets: human, information, organization. 
Strategy maps usage is typically aiming to establish a strategy to be achieved in the future; however, they may also be used for analyzing the status of a current business strategy striving for completeness. We classify the former as the to-be usage (i.e. perspective), while the latter as $a s$-is.

The to-be usage refers to a map representing a strategy for the future of an organization. It could be a strategy map for a newly established organization or a refinement of an existing strategy map being the result of having incorporated the BSC framework with specific objectives, measures and targets and results from the prior period have shown changes that need to be made. The best way to build a strategy map is to follow a top down manner, as suggested by [25], starting from a mission statement and core values to develop a strategic vision, which should project the organization's overall goal. Consequently, according to the template, all four perspectives need to be explored from the financial to the customer, to the internal to the learning and growth. In this category of strategy maps completeness is taken for granted because for an existing strategy map, completeness is essential, as no incomplete strategy map may exist. For a newly established strategy map completeness must be achieved, because the BSC framework cannot be incorporated into an incomplete strategy map and consequently any relevant action plan, as well as the fact that an incomplete strategy map is not a considered as a strategy map.

The $a s$-is usage refers to a map representing a current strategy of an organization. Such usage serves analysis purposes of current goals by migrating to strategy maps. An as-is map can be used to identify missing goals, processes and assets but may also be considered as an intermediate state for an existing organization that regards to adopt strategy maps for the first time. Such effort will require a few iterations taken in the same top-down manner described above. Such analysis purposes allow a strategy map to be in an incomplete state requiring further improvement, thus not being applicable to the BSC framework and any relevant action plan.

In the strategy map meta-model that follows, we have considered capturing both the $a s$-is and the to-be usage.

\subsection{The Strategy Map Meta-model}

According to [28], also echoed by [29], a meta-model defines the conceptual elements of a language as well as their possible interrelations. Contextual conditions, or static semantics, are constraints which we have defined through inference from the strategy map template and implementations found in the studied literature. For each language construct, constraints define the interrelations allowed and restrictions imposed.

The strategy map meta-model is presented in the figure below. The strategy map template [1] [15] constitutes the basis for the presented interrelating concepts, while additional conceptual variations of strategy map applications are embodied through constraints.

We present classes and interrelations of the meta-model by referring to the strategy map template and the relevant constraints by referring to applications that introduce conceptual variations.

The meta-model consists of 6 classes: the Strategy-map class, the Group class, the Perspective class, the Theme class, the Goal class and the Causality-relation class. Additionally we have introduced cardinality constraints for the relations between classes as we could infer them from the strategy map template and strategy map implementations found in the studied literature. 


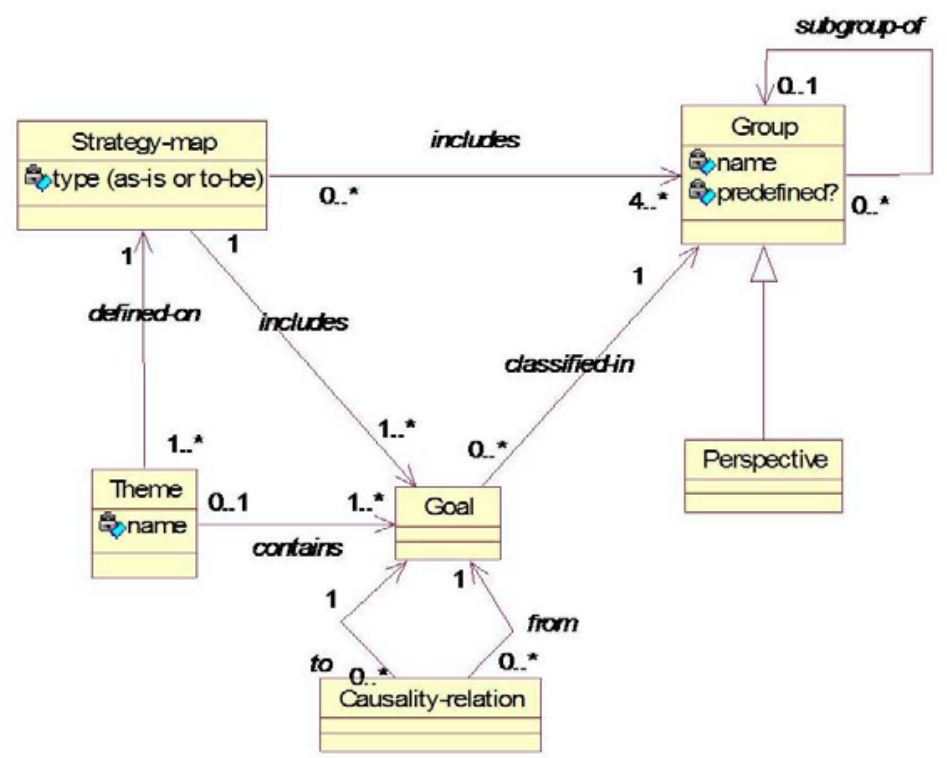

Fig. 2. The Strategy Map Meta-model

\subsubsection{Classes}

The Strategy-map class refers to the whole strategy map and holds a type attribute, which includes two possible values, either "as-is" or "to-be". "As-is" refers to a state where a strategy map is built to capture the current strategy of an enterprise for analysis purposes implying the possibility that the map is in an incomplete state requiring further improvement. "To-be" refers to a map representing a strategy for the future of the company. We here make the hypothesis that such a map must be complete and ready to incorporate the BSC framework with specific objectives, measures and targets. Our hypothesis is based on the fact that strategy maps are rooted on balanced scorecards (BSC), therefore, "balance" requires completeness. The distinction is made through the type attribute to distinguish between strategy maps built for analysis and strategy maps built for strategy.

The Goal class refers to goals set throughout the strategy map. Goals ${ }^{1}$ are defined and grouped within the financial and customer perspectives considering both the strategy map template as well as other applications of strategy maps [26]. For the internal perspective, as well as the learning and growth perspective we consider that both processes and capital appear in a strategy map in the form of goals. A process is executed to satisfy a goal [18] which though the cause-effect relation within a strategy map supports the goals at the customer perspective. In the same token, goals are set for all groups of capital referring to particular assets (in this context we imply goals

\footnotetext{
${ }^{1}$ The terms goal and objective are interchangeably used even within the sources referring to the same concept, a desired future state. However, given the fact that for the BSC framework objectives are measurable goals based upon which targets are defined, we use only the term goal within strategy maps, thus, the Goal class. Objectives are used when the balanced scoreboard is applied to a strategy map.
} 
on assets) aiming at desired competencies, capabilities needed to support, through the cause-effect relation within a strategy map, the internal processes.

The Causality-relation class refers to the cause-effect relation between goals within a strategy map and results into a tree structure of goals that when completed link goals on all perspectives.

The Group class refers to any grouping and any categorization of goals included in a strategy map (e.g. internal process clusters, capital, etc.) and holds two attributes, a name and a Boolean declaring whether the group is predefined (all groupings coming from the strategy map template are acknowledged as predefined). This class captures all possible groupings, the ones predefined in the strategy map template (e.g. internal processes, groups of capital, groups for the customer value proposition and groups within the financial perspective) while also allowing custom groupings to be introduced.

Sub-groups can be introduced into groups thereby making the nesting of groups inside other groups a tree. For example, "Operations Management Process" shown on the template is a sub-group of the "Internal Perspective" group.

The Perspective class refers to the highest level of grouping within a strategy map and is related to the Group class through generalization. Every strategy map includes the four predefined perspectives stated earlier.

The Theme class refers to the strategic theme(s) chosen within a strategy map. A strategic theme is a vertical slice within a strategy map that consists of a specific set of interrelated objectives. As discussed in [25] strategy is build top down, however, strategic themes are set by executives who identify the few critical processes (internal perspective) important for differentiating the customer value proposition (customer perspective) [24]. The vertical slice is then extended to all perspectives and their related goals are identified through the cause-effect relation.

\subsubsection{Constraints}

A number of constraints have been introduced to capture the variability of concepts found between the strategy map template and other strategy map applications. The following constraints apply to the meta-model:

- For the Strategy-map class:

- Every strategy map includes the four predefined perspectives of the strategy map template.

- If a strategy map is to-be, then every group that it includes and that itself has no subgroups included, must have at least one goal defined (it must be complete in the sense that goals must be defined in all categories).

- For the Group class:

○ If a group is a perspective, then it must not be a sub-group of another group. Perspectives constitute the highest level of grouping.

- If a group is not a perspective, then it is included in another group. A group that is not a perspective belongs at least to a perspective, or to another group.

- If a group is not predefined it must be included in at least one strategy map. New groups cannot be introduced unless they are used in a strategy map. 
- For the Goal class:

○ Every goal included in a theme is also included in the strategy map for which the theme is defined.

- For the Causality-relation class:

- A causality relation links two goals included in the same strategy map.

\section{Conclusions and Future Work}

In this study we have addressed the problem of aligning business strategies with operational aspects of enterprises. We have argued that for solving this problem the core need is to define a rich and well-defined language for modeling business strategies. Such a language can be further used for at least two purposes: (a) to serve as a reference for analyzing and comparing the capabilities of existing intentional modeling languages, both from the requirements engineering domain ( $i^{*}$, KAOS, GRL, etc.) and from the business domain (BMM, etc.), and (b) to facilitate transformations of high-level business notions related to stakeholder intensions and strategies to operational aspects, such as tasks and resources.

After a literature study, we have decided to consider the description of business strategies and the related notions proposed by Kaplan and Norton with strategy maps, as a relevant basis for ontology of business strategy. In this context, we have defined a well-structured and correct conceptualization (i.e. a meta-model) of strategy maps, and we have also elicited possible usages, which frame the scope of this study. We have outlined the meta-model by analyzing and interpreting numerous written sources on strategy maps and their utilizations. Our meta-model has included all major notions of strategy maps, such as the strategy perspectives, the containing groups of goals, as well as their causal relationships. The meta-model also supports the major utilization scenarios of strategy maps: (a) as-is, enabling modeling of current strategies for an enterprise aiming at analyzing them from a strategy map's requirements scope, and (b) modeling of to-be business strategies by following requirements for obtaining complete and correct strategy maps. Moreover, the metamodel generalizes some elements of the strategy map template such as the notion of group that covers both perspectives and lower level groups, while it introduces explicitly the notion of user defined perspectives and groups. Finally, it clearly separates classes and instances, where instances being contextually dependent, they are not explicitly shown but must be declared for an implementation.

The meta-model is aligned to the strategy map template as well as to the context dependent implementations found in the studied literature, inferring constraints from textually ambiguous descriptions. Therefore, our contribution is yet to be validated through case studies.

Future work concerns the extension of the meta-model to include the "realization" aspects of strategy maps using the BSC framework; consequently such a model will facilitate comprehensive evaluations of the well-known languages for intentional modeling from the requirements engineering and business domains such as $i^{*}, \mathrm{KAOS}$, BMM, etc. with respect to the concepts of business strategies as formalized in this paper on the basis of the strategy maps proposal. We also plan to design a formalization of 
strategy maps in an ontological form to provide a richer semantic basis when utilizing business strategies to be transformed to lower-level models, such as business process models. Our ontological base will also be gradually extended to facilitate more approaches for representing business strategies and consequently, the resulting model will also facilitate further comprehensive evaluations of goal-based languages.

\section{References}

1. Kaplan, R.S., Norton, D.P.: Strategy Maps: Converting Intangible Assets into Tangible Outcomes. Harvard Business School Press, Boston (2004)

2. Kaplan, R.S., Norton, D.P.: Mastering the Management System. J. Harvard business review 86, 63-77 (2008)

3. van Lamsweerde, A.: Goal-oriented requirements engineering: a guided tour. In: 5th IEEE International Symposium on Requirements Engineering, pp. 249-262. IEEE Press, New York (2001)

4. Yu, E.: Towards Modeling and reasoning Support for Early-Phase Requirements Engineering. In: 3rd IEEE International Symposium on Requirements Engineering (RE 1997), pp. 226-235. IEEE Press, Washington (1997)

5. Business Rules Group (BRG): The Business Motivation Model. Group (2007)

6. Anaya, V., Berio, G., Harzallah, M., Heymans, P., Matulevicius, R., Opdahl, A., Panetto, H., Verdecho, M.J.: The Unified Enterprise Modelling Language - Overview and further work. J. Computers in Industry 61 (2009)

7. Kavakli, E., Loucopoulos, P.: Goal Driven Requirements Engineering: Evaluation of Current Methods. In: 8th CAiSE/IFIP8.1 Workshop on Evaluation of Modeling Methods in Systems Analysis and Design, EMMSAD (2003)

8. Wilson, S.P., Kelly, T.P., McMermid, J.A.: Safety Case Development: Current Practice, Future Prospects. In: 1st ENCRESS/5th CSR Workshop (1995)

9. Basili, V.R., Rombach, D.D.: The TAME Project: Towards Improvement-Oriented Software Environments. J. IEEE Transactions on Software Engineering 14, 758-772 (1988)

10. Amyot, D., Ghanavati, S., Horkoff, J., Mussbacher, G., Peyton, L., Yu, E.: Evaluating goal models within the goal-oriented requirement language. J. Intelligent Systems 25, 841-877 (2010)

11. Chung, L., Nixon, B.A., Yu, E., Mylopoulos, J.: Non-Functional Requirements in Software Engineering. Kluwer Academic Publishers, Dordrecht (2000)

12. Popova, V., Sharpanskykh, A.: Formal Modelling of Goals in Organizations. Technical report, VU University faculty of Science (2008)

13. Camponovo, G.: Conceptual Models for Designing Information Systems Supporting the Strategic Analysis of Technology Environments. PhD Thesis. Information Systems Institute, HEC School of Business, University of Lausanne (2006)

14. Barney, J.: Types of Competition and the Theory of Strategy: Toward an Integrative Framework. J. Academy of Management Review 11, 791-800 (1986)

15. Kaplan, R.S., Norton, D.P.: The Balanced Scorecard: translating Strategy into Action. Harvard Business School Press, Boston (1996)

16. Bleistein, S.J., Cox, K., Verner, J.: Validating strategic alignment of organizational IT requirements using goal modeling and problem diagrams. J. Systems and Software 79, 362-378 (2006) 
17. Thevenet, L.H., Salinesi, C.: Aligning IS to organization's strategy: the INSTAL method. In: Krogstie, J., Opdahl, A.L., Sindre, G. (eds.) CAiSE 2007 and WES 2007. LNCS, vol. 4495, pp. 203-217. Springer, Heidelberg (2007)

18. Nurcan, S., Etien, A., Kaabi, R., Zoukar, I., Rolland, C.: A strategy driven business process modelling approach. J. Business Process Management 11, 628-649 (2005)

19. Gordijn, J., Petit, M., Wieringa, R.: Understanding business strategies of networked value constellations using goal- and value modeling. In: 14th IEEE International Requirements Engineering Conference (RE 2006), pp. 129-138 (2006)

20. van der Raadt, B., Gordijn, J., Yu, E.: Exploring web services ideas from a business value perspective. In: 13th IEEE International Conference on Requirements Engineering (RE 2005), pp. 53-62. IEEE CS, Los Alamitos (2005)

21. Edirisuriya, A.: Design Support for e-Commerce Information Systems using Goal, Business and Process Modelling. PhD Thesis. Department of Computer and Systems Sciences, Stockholm University (2009)

22. Singh, S.N., Woo, C.: Investigating business-IT alignment through multi-disciplinary goal concepts. J. Requirements Engineering 14, 177-207 (2009)

23. Babar, A., Zowghi, D., Chew, E.: Using Goals to Model Strategy Map for Business IT Alignment. In: $5^{\text {th }}$ International Workshop on Business/IT Alignment and Interoperability (BUSITAL 2010), pp. 16-30 (2010)

24. Kaplan, R.S., Norton, D.P.: The strategy map: guide to aligning intangible assets. J. Strategy \& Leadership 32, 10-17 (2004)

25. Kaplan, R.S., Norton, D.P.: Having trouble with your strategy? Then map it. J. Harvard Business Review 78, 167-176 (2000)

26. Olve, N.G., Petri, C.J., Roy, J., Roy, S.: Making Scorecards Actionable: Balancing Strategy and Control. John Wiley \& Sons Ltd., West Sussex (2003)

27. Cobbold, I., Lawrie, G.: The development of the Balanced Scorecard as a strategic management tool 2GC Conference Paper. In: PMA Conference, pp. 0-9 (2002)

28. Harel, D., Rumpe, B.: Meaningful Modeling: What' $s$ the Semantics of "semantics"? J. Computer 37, 64-72 (2004)

29. Lucena, M., Santos, E., Silva, C., Alencar, F., Silva, M.J., Castro, J.: Towards a unified metamodel for $i^{*}$. In: 2nd International Conference on Research Challenges in Information Science (RCIS 2008), pp. 237-246 (2008) 